\title{
A Review of Double Common Bile Duct and Its Sequelae
}

\author{
Sindhura Kollia, b, Denzil Etienne ${ }^{\mathrm{a}}$, Madhavi Reddy ${ }^{\mathrm{a}}$, \\ Ghulamullah Shahzad ${ }^{\mathrm{a}}$
}

\begin{abstract}
A double or accessory common bile duct (ACBD) is a rare congenital anomaly. We report the case of a 60-year-old American Asian male, who was found to have a double or duplicated common bile duct after being admitted for evaluation of a pancreatic mass. A duplicated bile duct has the same mucosa histologically as a single bile duct. However, the opening of a duplicated bile duct lacks a sphincter allowing retrograde flow of gut contents which results in a higher probability of intraductal calculus formation. On rare occasions, it can predispose to liver abscesses, pancreatitis, pancreatic cancer, gallbladder cancer, gastric cancer, and ampullary cancer depending on the location of the opening of the ACBD. We present an integrative review of the limited cases of ACBD with correlation to the current case and discussion regarding the aspects of diagnosis and management.
\end{abstract}

Keywords: Accessory common bile duct; Pancreatic cancer; Duplicated bile duct; Double common bile duct

\section{Introduction}

In 1543, Vesalius identified the anatomical insertion of the common bile duct into the duodenum [1]. In the 500 years since then, the anatomical deviation of the duct into a double common bile duct has been an increasingly rare incidence with Telium [2] identifying only 24 cases in Western literature until the year 1986 [3]. Yamashita subsequently recorded 47 cases in Japanese literature from 1968 to 2002, revealing an incidence that favored an Oriental population. Among the 47 cases observed by Yamashita, it was noted that the age of the patients ranged from four days after birth to 80 years old, with a mean age of 46.1 years old. The ratio of female to male patients was 1.6:1. The chief symptoms were epigastric or right

Manuscript submitted December 15, 2017, accepted December 20, 2017

aDepartment of Gastroenterology and Hepatology, The Brooklyn Hospital Center, Academic Affiliate of The Icahn School of Medicine at Mount Sinai, Clinical Affiliate of The Mount Sinai Hospital, Brooklyn, NY, USA

${ }^{b}$ Corresponding Auther: Sindhura Kolli, Department of Gastroenterology and Hepatology, The Brooklyn Hospital Center, Academic Affiliate of The Icahn School of Medicine at Mount Sinai, Clinical Affiliate of The Mount Sinai Hospital, 121 Dekalb Avenue, Brooklyn, NY 11201, USA.

Email: sindhura.kolli@gmail.com

doi: https://doi.org/10.14740/gr950w hypochondrium quadrant pain in 38 cases (80.1\%). Clinical manifestations were found to be a result of inflammation in and around the biliary system; pancreatitis was present in five cases $(10.6 \%)$, cholangitis in five cases $(10.6 \%)$, cholecystitis in two cases $(4.3 \%)$, and liver abscess in one case $(2.1 \%)$. Cholelithiasis was found in 13 cases $(27.7 \%)$, a choledochal cyst in five cases $(10.6 \%)$, pancreaticobiliary maljunction (PBM) in 14 cases (29.8\%), and upper gastrointestinal cancers such as gallbladder cancer, gastric cancer, ampullary cancer and pancreatic cancer in 12 cases $(25.5 \%)$. Five patients died from progression of these primary cancers [4].

\section{Literature Search}

PubMed and Google Scholar were used to perform a systematic review of the available literature. Searches using the following keywords "double common bile duct", "duplicated common bile duct", "accessory common bile duct", and "pancreatic cancer", were performed from 2002 to 2016.

\section{Case Description}

A 60-year-old Middle Eastern male patient with a past medical history of benign prostatic hyperplasia, hypertension, gastroesophageal reflux disease and type 2 diabetes mellitus presented to the emergency department complaining of crampy lower abdominal pain associated with an unintentional 40 pounds weight loss over 4 months. A magnetic resonance imaging (MRI) of the abdomen with intravenous contrast demonstrated a heterogeneous solid pancreatic uncinate mass $(3.9 \times 3.2 \mathrm{~cm})$ with a duplicated common bile duct (Fig. 1).

A subsequent endoscopic ultrasound (EUS) confirmed a large heterogeneous hypoechoic mass with irregular borders and cystic areas in the uncinate process of the pancreas. The distal CBD appeared mildly dilated with two extrahepatic bile ducts consistent with type II accessory common bile duct (ACBD). The accessory bile duct provided a conduit for the deposition of bile in the pancreatic head where it terminated. Subsequent inflammation progressed to metaplastic changes confirmed by a fine needle aspiration (FNA) via trangastric approach. The atypical cells of metaplasia found in the pancreatic head were confirmed to be pancreatic adenocarcinoma. Magnetic resonance cholangiopancreatography (MRCP) confirmed the above EUS findings along with multiple enhancing foci also present in the liver. A triple phase computerized tomography (CT) also re-demonstrated an ACBD (Fig. 2) with 


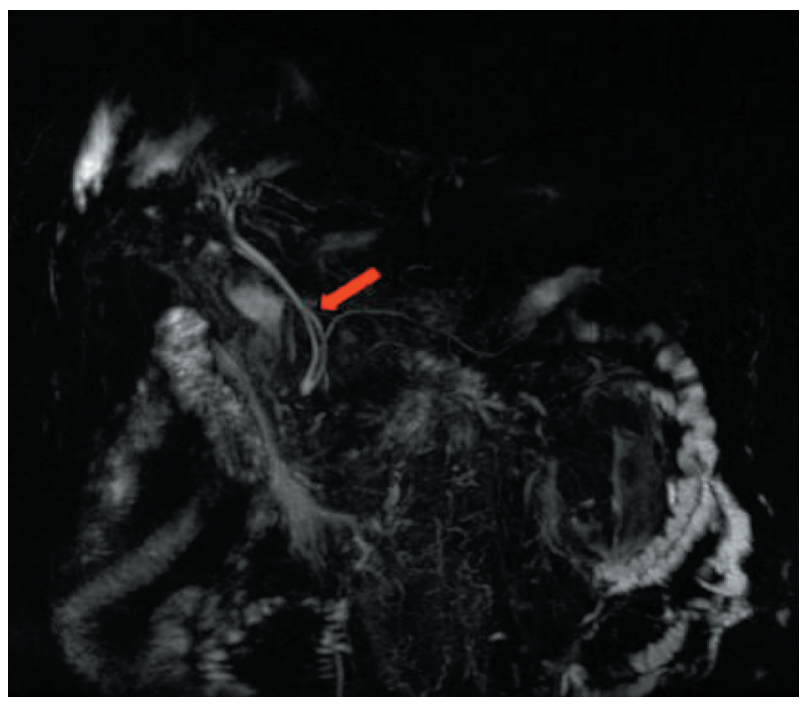

Figure 1. Magnetic resonance imaging (MRI) depiction of ACBD.

multiple hypervascular hepatic lesions and a soft tissue mass in the pancreatic uncinate process (Fig. 3) encasing the superior mesenteric artery (SMA). The patient was eventually discharged for further staging and subsequent chemotherapy at a different institution.

\section{Integrative Review}

An ACBD arises embryologically in the third week of gestation. As the liver and biliary tree develop, the stem of the hepatic primordium becomes the bile duct and its lumen is developed by recanalization of the epithelium. The development of double common bile duct can be ascribed to disturbances in recanalization of the hepatic primordium [5] with the accessory bile duct opening into a part of the upper gastrointestinal tract (stomach, duodenum, ductus pancreaticus or septum).

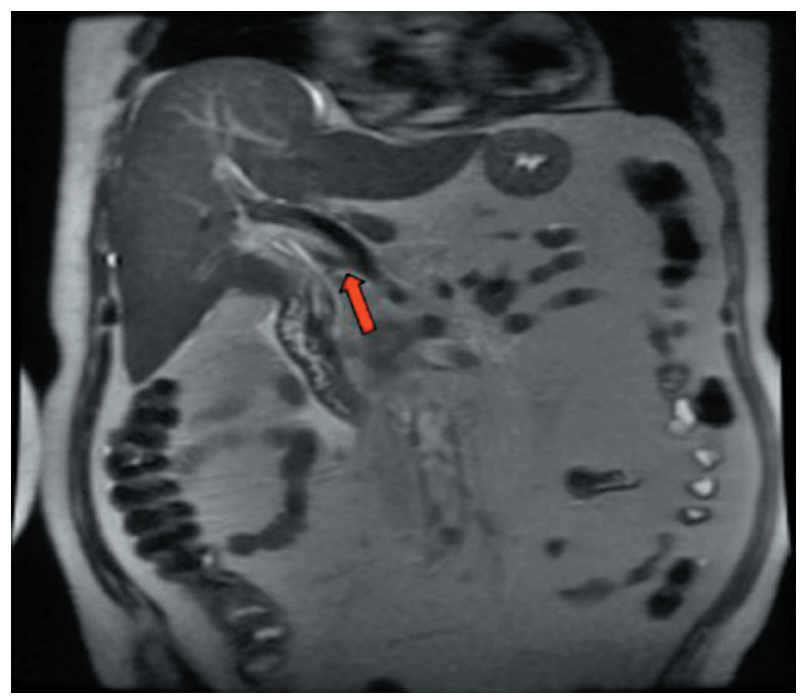

Figure 2. ACBD on CT imaging.

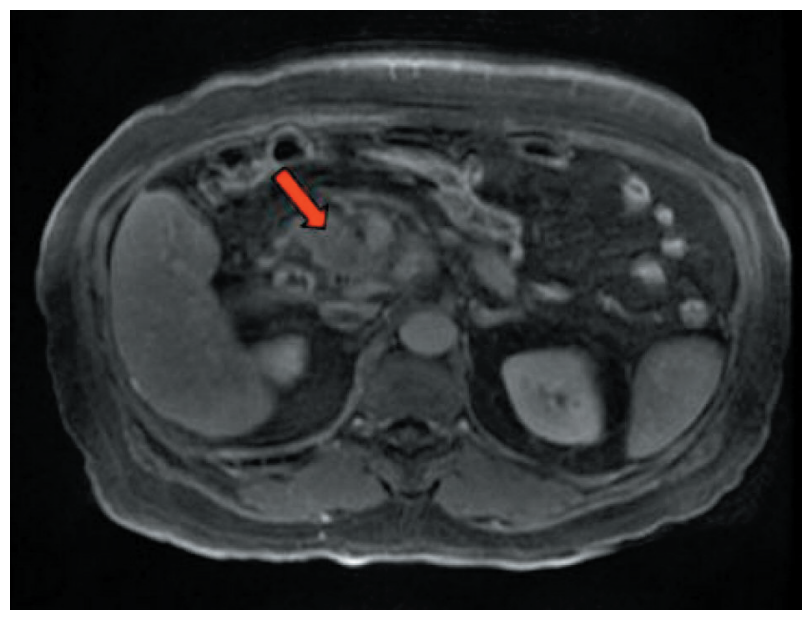

Figure 3. CT imaging of pancreatic cancer.

A duplicated bile duct has the same mucosa histologically as a single bile duct. However, the opening of a duplicated bile duct lacks a sphincter allowing retrograde flow of gut contents which results in a higher probability of intraductal calculus formation [6].

The earliest classification of double common bile duct was developed by Goor and Ebert (1972) [7] and was based on anatomical structure. It was later modified by Saito [8] in 1988, who classified it without regard to the anatomical opening of the ACBD. However, we currently follow the classification based on morphology, proposed by Choi in 2005 [9], who described additional variants. In the description by Choi, five types of accessory common bile ducts exist. Type I: septum dividing the bile duct lumen, II: bifurcation of the distal bile duct and each channel draining independently into the bowel, III: duplicated extrahepatic bile ducts (a) without or (b) with intrahepatic communicating channels, IV: duplicated extrahepatic bile duct with extrahepatic communicating channel or more than one communicating channels, $\mathrm{V}$ : single biliary drainage of double bile ducts without (a) or with (b) communicating channels (Fig. 4) [9]. The most commonly reported are types III or IV with one duct opening into the major duodenal papilla and a second duct (often referred to as accessory bile duct or
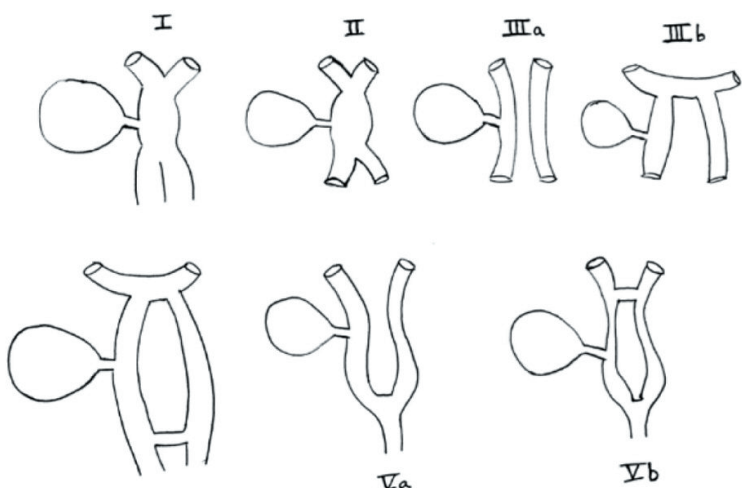

IV

Figure 4. Original depiction of Choi classification of types of ACBD [9]. 
ACBD) opening into the stomach, duodenum, or pancreatic duct. The least common is type $\mathrm{V}$ with only one case each of type $\mathrm{Va}$ and $\mathrm{Vb}$ having been reported previously [10].

Precise preoperative recognition is crucial. If the condition is detected preoperatively, careful dissection combined with intraoperative cholangiography can avoid misidentification of the anatomy and inadvertent biliary injury or accidental ligation of a low riding cystic duct, especially during laparoscopic cholecystectomy [10]. Detecting an ACBD is most commonly performed via an EUS. Non-invasive alternatives are MRCP and contrast CT, while the current gold standard is an endoscopic retrograde cholangiopancreatography (ERCP). Because an ERCP is an operator-dependent, more invasive technique that carries significant complications, including pancreatitis and duodenal perforation, MRCP is usually preferred in the screening of biliary malignancies in high-risk patients with pancreaticobiliary malformations. The proximal location of the sphincter of Oddi relative to the pancreaticobiliary junction is believed to result in the continuous regurgitation of pancreatic juices into the bile duct, predisposing it to malignant change. In such cases, examination of the biliary tree with an MRCP may be helpful in corroborating other associated congenital abnormalities, as well as evaluating any pathological change [8]. However, in instances when the duct is obstructed with stones or has communicating channels that must be visualized, a multi-detector row computed tomography (MDCT) using the minimum intensity projection (MinIP) technique enables radiologists to acquire a large volume of images rapidly thus facilitating high spatial resolution imaging of the biliary tree [11]. This technique has been shown to be especially useful in detecting type $\mathrm{Vb}$ ACBD which has connecting channels or other ACBD types with multiple stone obstructions.

Symptoms of ACBD include epigastric pain, nausea or vomiting, right upper quadrant pain, heartburn, fever, and jaundice, though it may also be asymptomatic [12]. According to Yamashita's review [4], gallbladder cancer, gastric cancer, ampullary cancer and pancreatic cancer developed more frequently in ACBD patients. Gastric cancers developed only in patients with ACBD opening into the stomach, possibly due to prolonged exposure of the gastric mucosa to components of duodenal and pancreaticobiliary juice causing atrophic gastritis and serving as a nidus for the development of gastric cancer. Mason and Filipe demonstrated that bile reflux alone did not produce tumors, but that pancreatic and combined pancreaticobiliary reflux produced gastric cancer in animal studies [13]. Gallbladder cancer and ampullary cancer developed in patients with ACBD openings into the second portion of the duodenum, pancreatic duct and concomitant anomalous pancreaticobiliary ductal union (APBDU). APBDU has never been seen in cancer patients with ACBD openings into the first portion of the duodenum or stomach [14].

Treatment options vary depending on the presence of malignancy. In instances without gastric cancer, but in the presence of unremitting symptoms, surgical resection of the ACBD was recommended resulting in separation of the flow of bile and pancreatic juice into the gastrointestinal tract. If cancer is not present or if the ACBD is incidentally detected, an endoscopic biopsy of the surrounding gastric mucosa is recom- mended. If gastric mucosal dysplasia is present, a gastrectomy should be performed. If gastric mucosa biopsy returns negative for dysplasia, periodic endoscopic examination is recommended due to the potential increased risk of cancer [12]. Komi found bile duct carcinoma in $15.6 \%$ of adult cases and recommended surgical treatment to stop reflux of pancreatic juice into the biliary duct, citing pancreatic juice regurgitation into the biliary system as cause for carcinogenesis [14]. The prognosis of an ACBD is attributed to the concomitant pathologies, ranging from cholelithiasis to upper gastrointestinal cancers, so outcomes are often variably dependent on the sequelae, timely diagnosis and intervention [4].

\section{Author Contributions}

The above authors contributed equally and significantly to the abstract, introduction, methods, results, and discussion sections in the above titled review paper.

\section{Conflict of Interest}

None.

\section{References}

1. Lee JH, Yu JS, Park MS, Yoon DS, Yang SW. MR cholangiography of accessory bile duct connected to the stomach. AJR Am J Roentgenol. 2007;189(6):W344-347.

2. Teilum D. Double common bile duct. Case report and review. Endoscopy. 1986;18(4):159-161.

3. Park SW, Koh H, Oh JT, Han SJ, Kim S. Relationship between anomalous pancreaticobiliary ductal union and pathologic inflammation of bile duct in choledochal cyst. Pediatr Gastroenterol Hepatol Nutr. 2014;17(3):170-177.

4. Yamashita K, Oka Y, Urakami A, Iwamoto S, Tsunoda T, Eto T. Double common bile duct: a case report and a review of the Japanese literature. Surgery. 2002;131(6):676681.

5. Park MS, Kim BC, Kim T, Kim MJ, Kim KW. Double common bile duct: curved-planar reformatted computed tomography (CT) and gadobenate dimeglumine-enhanced MR cholangiography. J Magn Reson Imaging. 2008;27(1):209-211.

6. Djuranovic SP, Ugljesic MB, Mijalkovic NS, Korneti VA, Kovacevic NV, Alempijevic TM, Radulovic SV, et al. Double common bile duct: a case report. World J Gastroenterol. 2007;13(27):3770-3772.

7. Goor DA, Ebert PA. Anomalies of the biliary tree. Report of a repair of an accessory bile duct and review of the literature. Arch Surg. 1972;104(3):302-309.

8. Saito N, Nakano A, Arase M, Hiraoka T. [A case of duplication of the common bile duct with anomaly of the intrahepatic bile duct]. Nihon Geka Gakkai Zasshi. 1988;89(8):1296-1301.

9. Choi E, Byun JH, Park BJ, Lee MG. Duplication of the 
extrahepatic bile duct with anomalous union of the pancreaticobiliary ductal system revealed by MR cholangiopancreatography. Br J Radiol. 2007;80(955):e150-154.

10. Gupta V, Chandra A. Duplication of the extrahepatic bile duct. Congenit Anom (Kyoto). 2012;52(3):176-178.

11. Kim SW, Park DH, Shin HC, Kim IY, Park SH, Jung EJ, Kim CH. Duplication of the extrahepatic bile duct in association with choledocholithiasis as depicted by MDCT. Korean J Radiol. 2008;9(6):550-554.

12. Park JI, Oh SH. Double common bile duct with an ec- topic drainage into the stomach. Ann Surg Treat Res. 2015;88(4):229-231.

13. Mason R, Filipe I. The aetiology of gastric stump carcinoma in the rat. Scand J Gastroenterol. 1990;25(10):961965.

14. Komi N, Tamura T, Miyoshi Y, Kunitomo K, Udaka H, Takehara H. Nationwide survey of cases of choledochal cyst. Analysis of coexistent anomalies, complications and surgical treatment in 645 cases. Surg Gastroenterol. 1984;3(2):69-73. 